\title{
Sporozoon tincae infection in tench. An ultrastructural investigation
}

\author{
F.-J. Kaup ${ }^{1, *}$, W. Körting ${ }^{2}$ \\ ${ }^{1}$ Institute of Pathology, ${ }^{2}$ Fish Diseases Research Unit, School of Veterinary Medicine, Bünteweg 17, D-30559 Hannover, \\ Germany
}

\begin{abstract}
For several years now, pathological skin changes have been observed in tench Tinca tinca L. from northern Germany. Using light microscopy, numerous instances of the causative agent have been detected, cell-bound in macrophages and/or between altered tissue structures. The infection shows systemic spread, as the microorganisms, Sporozoon tincae, also appear in the spleen. Using electron microscopy, it was possible to depict numerous different features of the microorganism associated with the intracellular development and multiplication of the agent. The structures lacked a true cell nucleus, so the previous assignment of the organism to the Protozoa is no longer maintainable. Our findings demonstrate that $S$. tincae is not a eukaryotic organism. We suggest that $S$. tincae be classified as bacteria.
\end{abstract}

KEY WORDS: Sporozoon tincae $\cdot$ Tench $\cdot$ Tinca tinca $\cdot$ Electron microscopy

\section{INTRODUCTION}

Tench Tinca tinca L. belong to the group of prized, edible fish that prefer standing waters with a muddy bottom. Frequently they are bred together with carp in earth ponds. Since 1980, tench collected from northern Germany have shown multiple ulcers at the base of the fins, on the side of the body and in the region of the tail (Körting et al. 1987). The alterations were associated with parasites which were first described by Volf \& Dvořák (1928). The disease ascribed to Sporozoon tincae occurred in southern Bohemia (present Czech Republic). The symptoms and the light microscopy of the causative agent were redescribed by Jirovec et al. (1947). In contrast to the disease in Bohemia which, with a mortality rate of up to $90 \%$, reflects a widespread condition, those instances reported from northern Germany were only individual cases, which occurred regularly in the autopsy mater-

\footnotetext{
-Address for correspondence: Experimental Pathology, German Primate Centre (DPZ), Kellnerweg 4, D-37077 Göttingen, Germany
}

ial. From an electron microscopy study, it became clear that an unequivocal assignment to the Protozoa or to other eukaryotic organisms was not possible (Körting et al. 1987). The present study describes the morphology of the disease pattern and documents ultrastructural characteristics of its agent, information which until now has not been available in the published literature.

\section{MATERIAL AND METHODS}

The present study results from the investigation of 5 adult tench ( 3 to $4 \mathrm{yr}$ old) collected in the autumn from a carp farm in northern Germany. Following macroscopic inspection, samples were obtained from affected skin regions and from spleen immediately after sacrifice. The material was fixed with $10 \%$ buffered neutral formaldehyde for light microscopy and conventionally embedded in paraplast. The following staining techniques were carried out on paraffin sections: H\&E, PAS, Grocott, Giemsa, Ladewig, Azan. For electron microscopy, tissue samples were cut into blocks of approximately $1 \mathrm{~mm}^{3}$ 


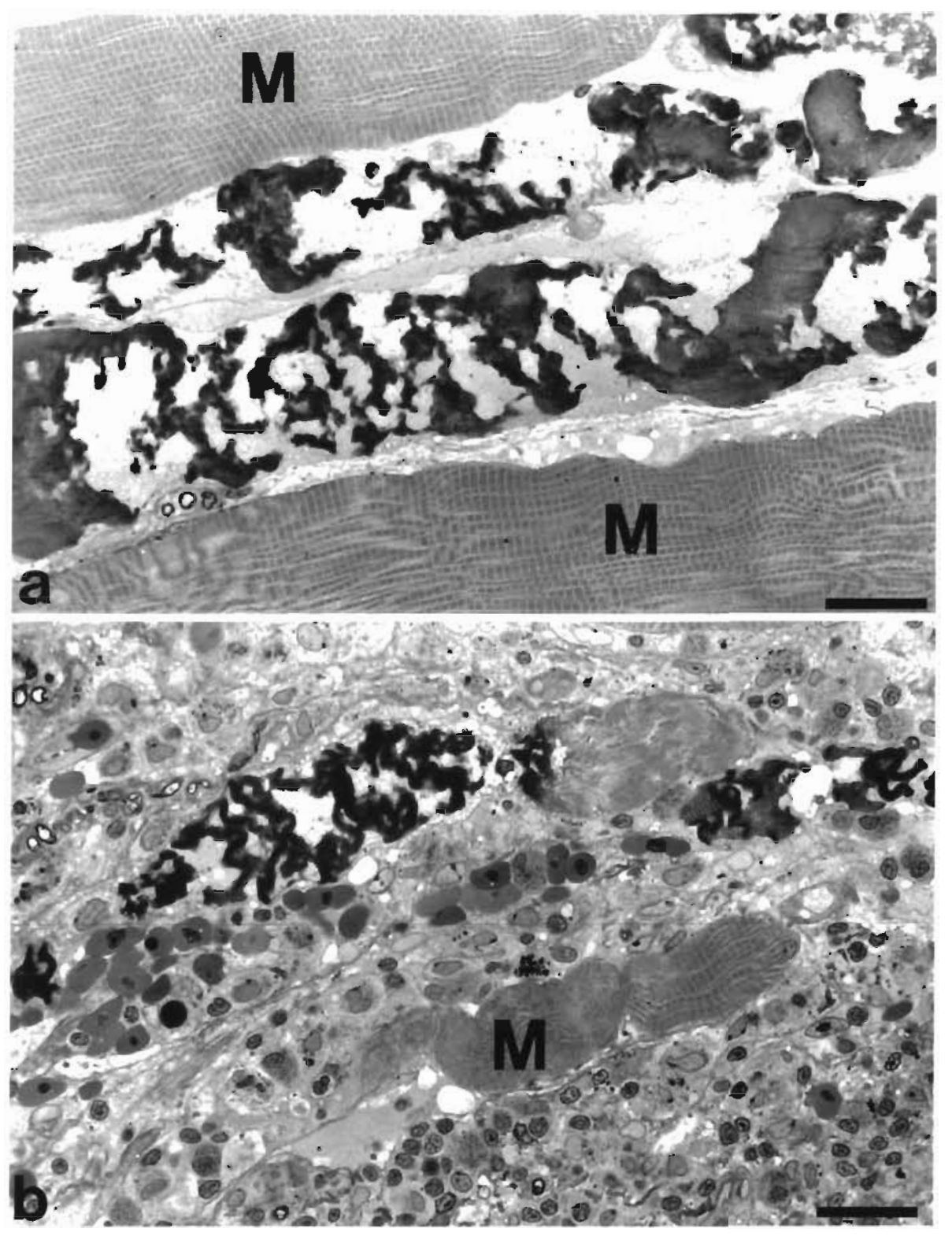

Fig. 1 Sporozoon tincae infecting Tinca tinca. Light micrographs demonstrating (a) severe hyaline degeneration of muscle cells (M), which is occasionally associated with (b) inflammatory cell infiltrates. Semithin section, toluidine blue, scale bars $=20 \mu m$

and fixed for $24 \mathrm{~h}$ in $2.5 \%$ cacodylate buffered glutaraldehyde. Blocks were postfixed in $1 \%$ osmium tetroxide buffered in $0.15 \mathrm{M}$ sodium cacodylate, repeatedly rinsed in cacodylate buffer, dehydrated in increasing concentrations of alcohol, and embedded in Epon 812 with propylenoxide as intermedium.
Toluidine-blue-stained semithin sections were used for light microscopic orientation. Ultrathin sections were automatically (Leica ultrostainer) contrasted with uranyl acetate $\left(30 \mathrm{~min}, 40^{\circ} \mathrm{C}\right)$ and lead citrate $\left(72 \mathrm{~s}, 20^{\circ} \mathrm{C}\right)$, and examined with a Zeiss EM $10 \mathrm{C}$ at $60 \mathrm{kV}$.

Fig. 2. Sporozoon tincae infectung Tinca tinca. Numerous microorganisms as punctiform structures are observed either extracellularly [(a) intraepithelial, (b) subepithelial] or (c) intracellularly in macrophages. E: epithelium of the skin. Semithin section, toluidine blue, scale bars $=(a, b) 20 \mu \mathrm{m}$, (c) $15 \mu \mathrm{m}$ 


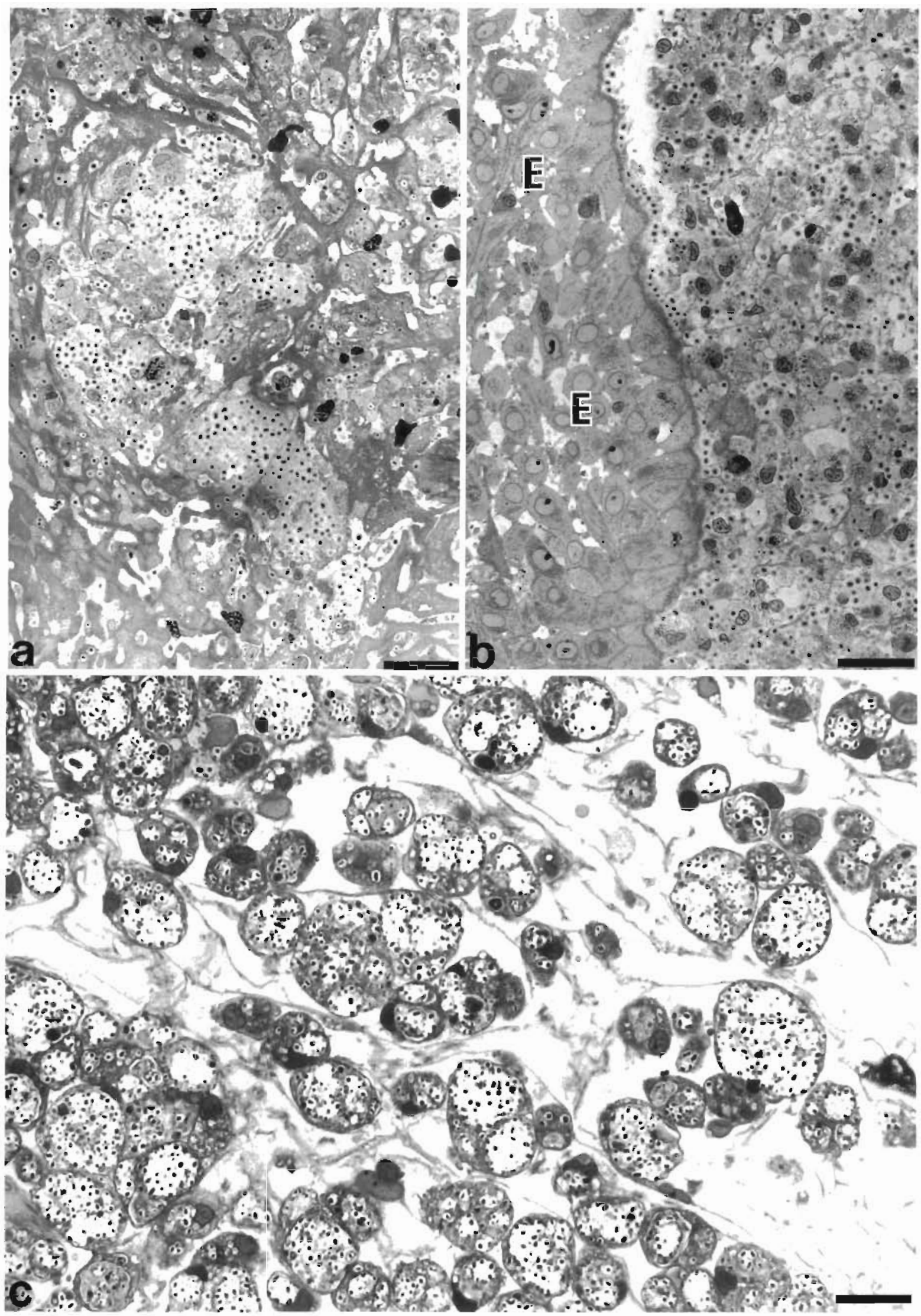




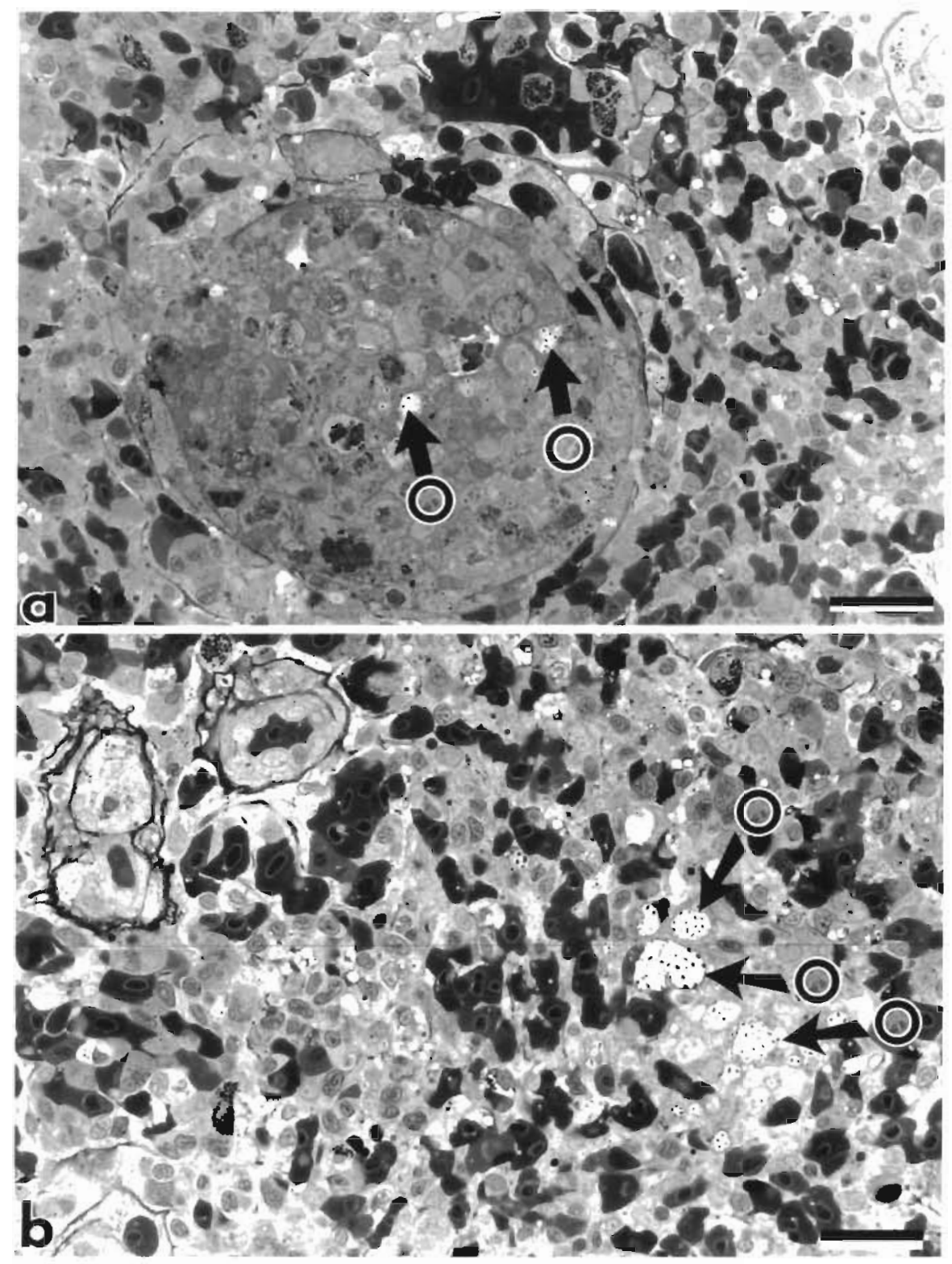

Fig. 3. Sporozoon tincae infectung Tinca tinca. ( $(\mathrm{a}, \mathrm{b}) \mathrm{S}$. tincae (O) within the splenic tissue. (a) In addition to the microorganisms, a granuloma with pigment-storing cells occurs. Semithin section, toluidine blue, scale bars $=20 \mu \mathrm{m}$

\section{RESULTS}

\section{Gross pathology}

Skin changes were seen in all 5 fish, predominantly at the base of the fins and the ventrolateral trunk region. In 2 tench, adcitional lesions were observed on the gills. One tench also showed such changes at the corners of the mouth. Furthermore, circumscribed subcutaneous haemorrhages or whitish shimmering areas (diameter up to $2 \mathrm{~cm}$ ) occurred in different regions of the skin. The whitish areas were detached from the body surface and exhibited haemorrhagic borders. In distinct cases, ulceration was present. Evaluation of the spleen revealed a picture ranging from unchanged to moderate swelling due to increased blood filling

\section{Histopathological lesions}

In affected regions of the skin, there were different histological changes such as necrosis, haemorrhages and inflammatory cell infiltrations. These processes also took hold of the adjacent muscle layers below and 


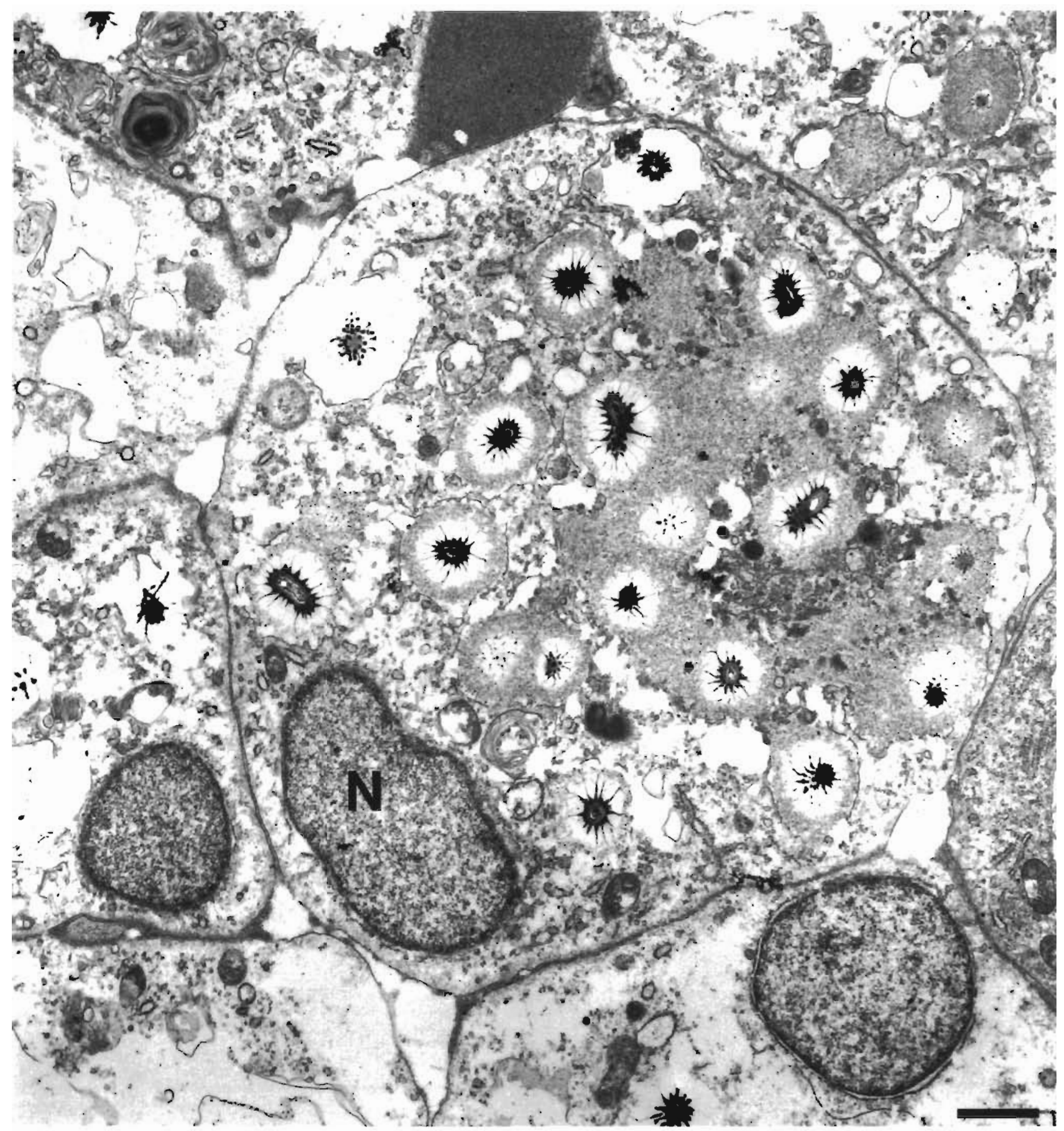

Fig. 4. Sporozoon tincae infecting Tinca tinca. Intracellular accumulation of $S$ tincae in a degenerating cell (N. nucleus) resembling the ultrastructure of a macrophage Transmission electron microscopy, scale bar $=1 \mu \mathrm{m}$

caused varying degrees of hypercontraction and hyaline degeneration (Fig. 1a, b). In 2 of the affected fish. the changes - particularly in the region of the base of the fins and on the tail fin - were accompanied by the appearance of numerous macrophages. Intracellularly, these macrophages revealed vacuoles containing the causative agent (Fig. 2c). The affected cells lay either closely packed in clusters or were located indıvidually in the tissues. The cell-bound microorganisms were observed in vacuoles that showed signs of confluence. In addition to the intracytoplasmic parasites, numerous ones also lay free in the tissue (Fig. 2a, b) This was 


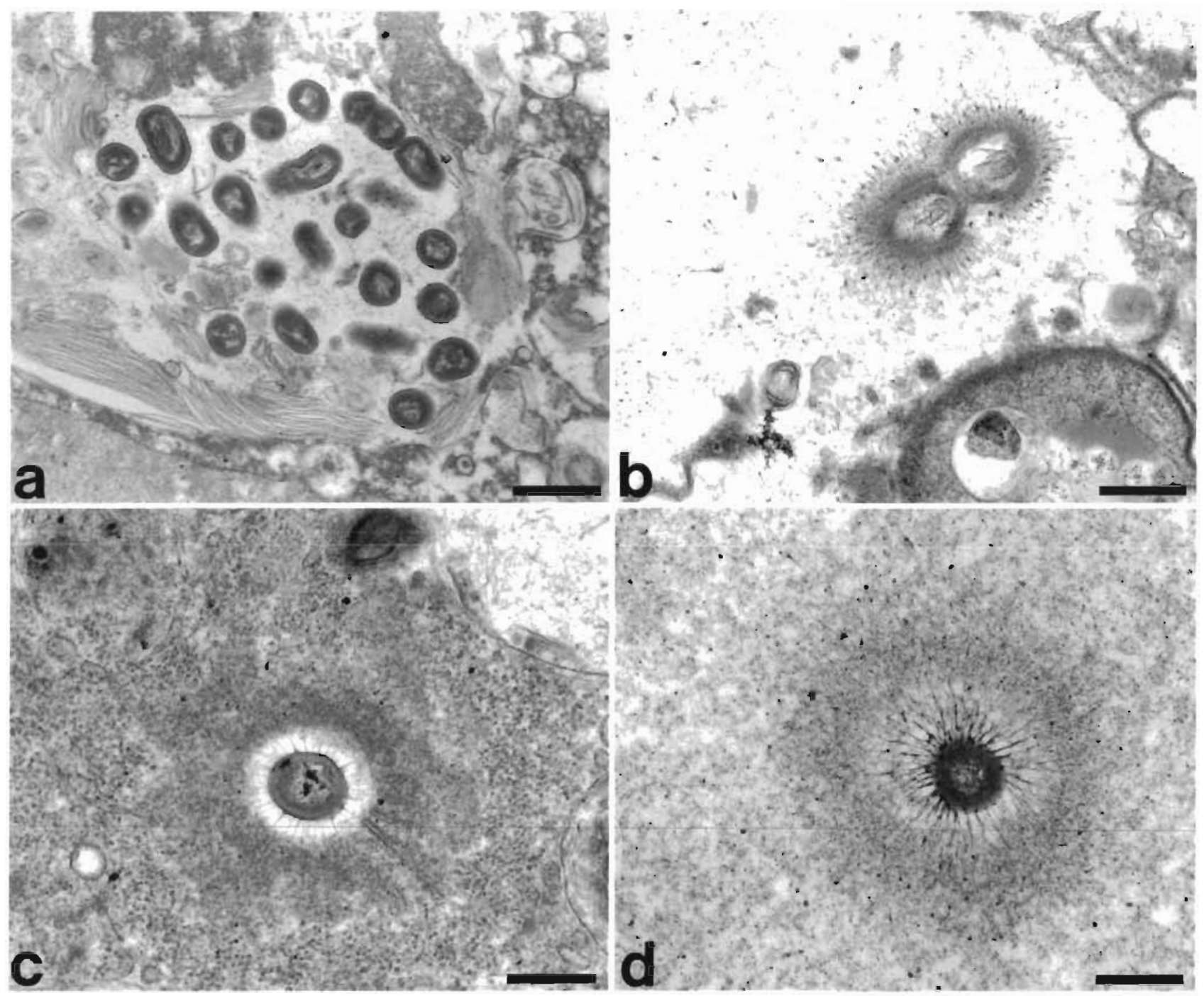

Fig. 5. Sporozoon tincae infecting Tinca tinca. Electron micrographs demonstrating various stages of proposed early development: the central structure consists of an electron-lucent centre and a peripheral dense membrane-bound border. The ultrastruc-

ture is similar to bacterial morphology. Signs of division are shown in (b). Scale bars = (a) $0.8 \mu \mathrm{m}$, (b, c) $0.5 \mu \mathrm{m}$, (d) $0.4 \mu \mathrm{m}$

observed particularly in areas with severe cell necrosis. Light microscopy revealed 2 features of the microorganims in semithin sections: in addition to dark points with a light halo, there were point-like structures with dark margins, which resembled fried eggs. Using Giemsa staining, the microorganisms stained strongly red-violet. PAS reaction was weakly positive, Grocott staining revealed negative results.

In the spleen of both fish showing cutaneous parasitic infection, the microorganisms could likewise be observed. In contrast to the skin lesions, the parasites in smaller numbers were only located intracellularly (Fig. 3a, b). Furthermore, accumulations of pigment-storing (probably hemosiderin) cells occurred (Fig. 3a).

\section{Transmission electron microscopy}

Ultrastructurally, there were different images of the parasites that lay in macrophages (Fig. 4). The microorganisms showed a central rod-like structure which was circular in cross section. The dimensions of the central structure ranged up to $1 \times 0.3 \mu \mathrm{m}$. The central structure presented an outer membrane, a peripheral electron-dense border and an electron-lucent, granular centre (Fig. 5a to d). The intracellular microorganisms were observed as single structures in the unchanged cytoplasm or in cytoplasmic vacuoles of different size and shape. Due to the confluence of these vacuoles, large intracytoplasmic cyst-like structures resulted which contained numerous microorgan- 


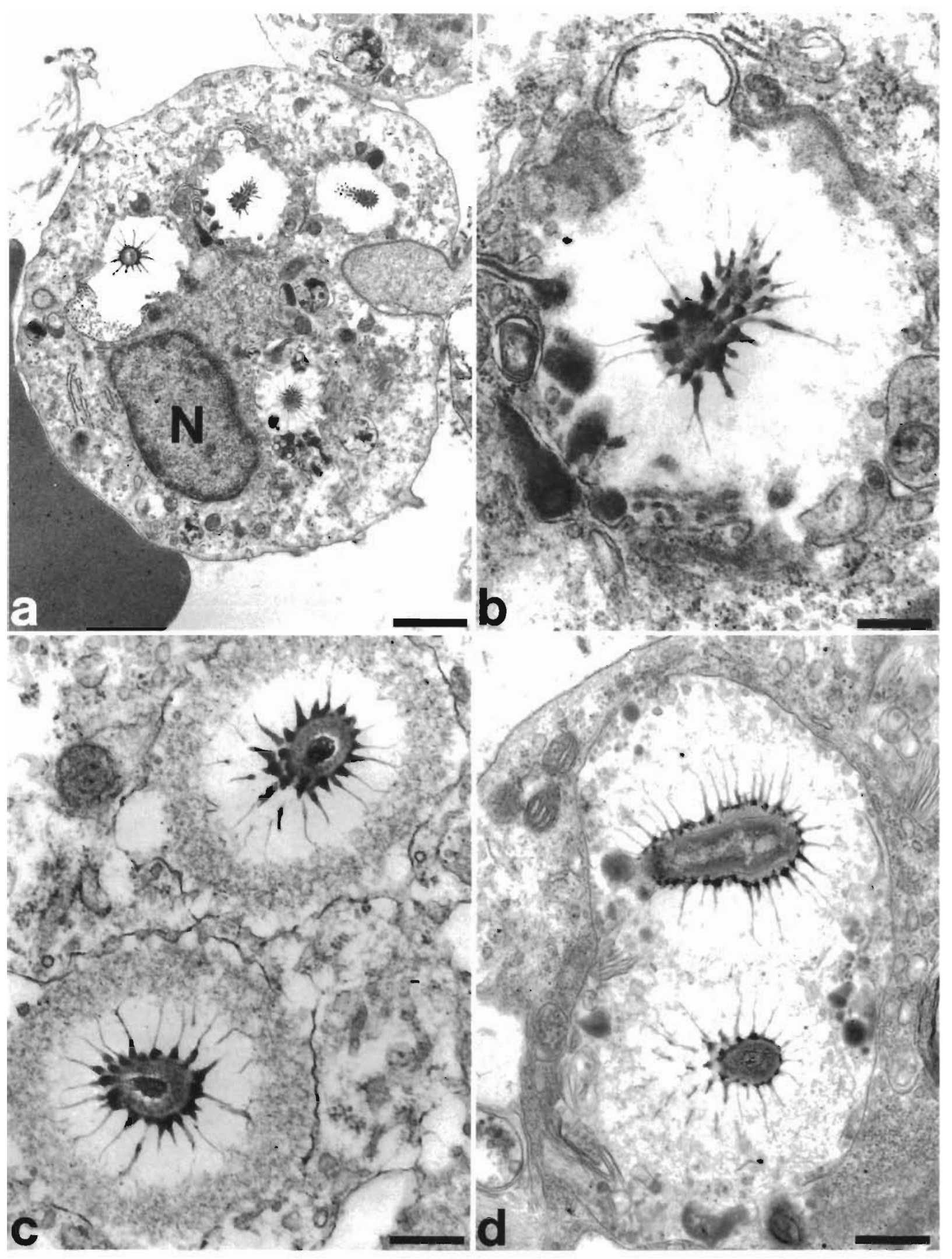

Fig. 6. Sporozoon tincae infecting Tinca tunca Intracytoplasmic vacuoles with mature structures of $S$ thncae (a) Survey of an infected cell (N: nucleus). Scale bar $=12 \mathrm{\mu m}$ (b) Tangential and longitudinal section of 1 microorganism Scale bar $=330 \mathrm{~nm}$ (c) Cross section of 2 vacuoles with $S$ tincae Scale bar $=330 \mathrm{~nm}$. (d) Confluence of 2 vacuoles Scale bar $=350 \mathrm{~nm}$ 


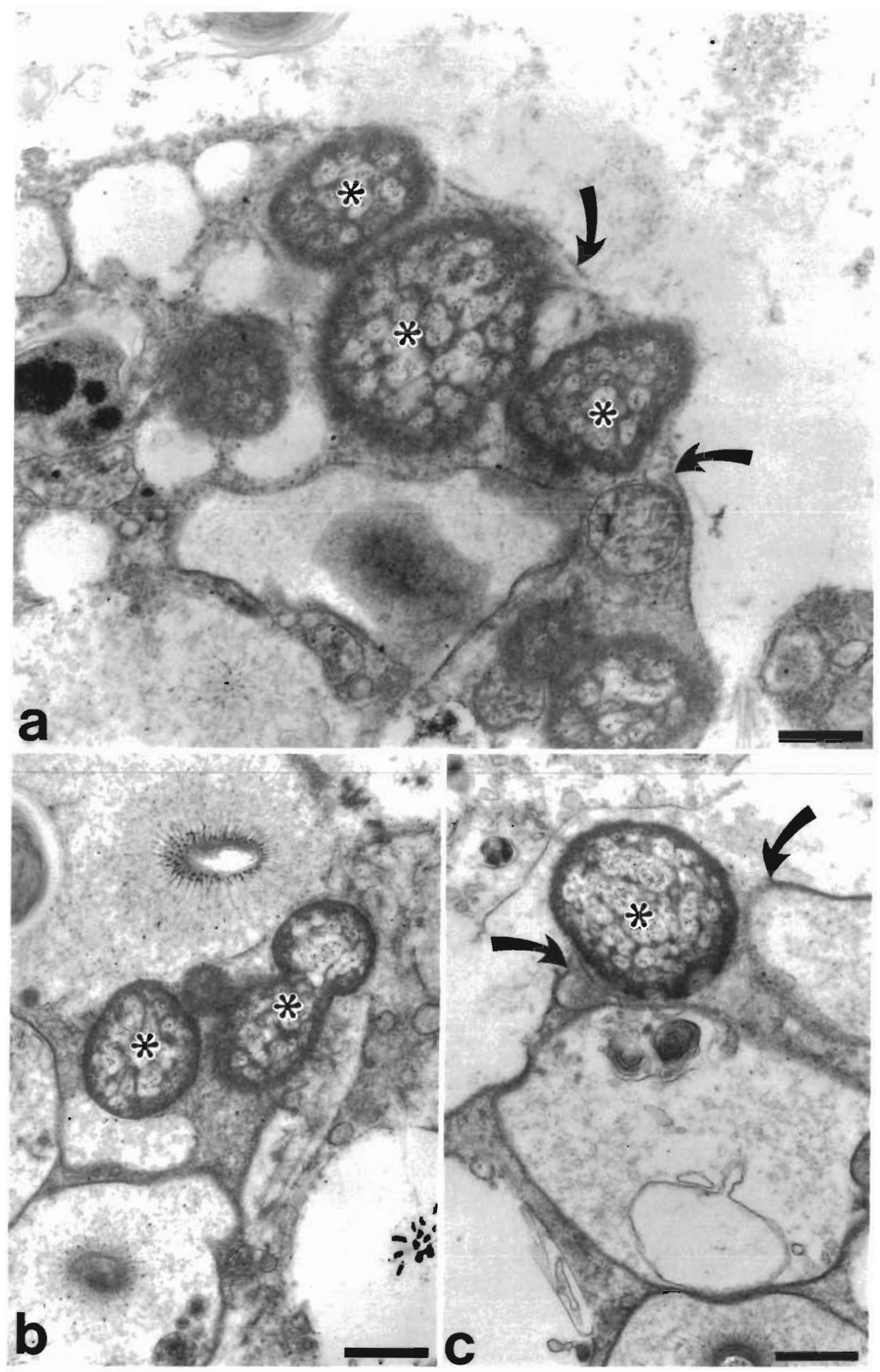

Fig 7. Sporozoon tincae infecting Tinca tinca. Intracytoplasmic, ovold, compartmentalised bodies ( $*$ ) of unknown origin, extruded to the surface of the microorganisms (arrows). Electron micrograph, scale bars = (a) $470 \mathrm{~nm},(\mathrm{~b}) 860 \mathrm{~nm},(\mathrm{c}) 570 \mathrm{~nm}$ 
isms. The membranes of the vacuoles or cysts were frequently only fragmentary. The affected macrophages revealed various signs of degeneration. Cell necrosis was accompanied by the extracellular release of microorganisms

In addition to the central structure, the vacuoles contained a fine, granular material or presented an electron-lucent space which was permeated by radial filaments drawing towards the centre (Fig. 6a to d). In cross section, these filaments had a star-shaped appearance and were inserted in the membranes of the central structure (Fig. 6c). In tangential sections, one could recognize that these attachment points were arranged in rows on the long side of the central, rodlike agent (Fig. 6b). Occasionally, it was possible to detect a division of the central rods (Fig. $5 b$ ).

In several affected cells, in addition to the described structures, intracytoplasmic formations were found which were round to ovoid, with a diameter of about $0.5 \mu \mathrm{m}$, and partly extruded (Fig. 7 a to $\mathrm{c}$ ). These structures possessed an electron-dense shell and a compartmentalised centre.

\section{DISCUSSION}

Following the first report by Volf \& Dvoŕák (1928), Sporozoon tincae was described in detail by Jirovec et al. (1947). The comparison of these descriptions with our results give us reason to believe that the same causative microorganisms are involved. In contrast to our cases, the previously mentioned authors observed a mortality of about $90 \%$ or more, while the infection and mortality rates in our cases were negligible (less than 1\%) (Körting et al. 1987). To our knowledge, there are no further reports concerning the occurrence of $S$. tincae. Whether all observed microscopic changes are due to $S$. tincae is not unequivocal as, despite pathohistological alterations, evidence of the causative agent was seen in only 2 cases. The contribution of other causes to the pathogenesis of the skin lesions is therefore possible (Körting et al. 1994). The occurrence of infected macrophages in the spleen indicates the systemic character of the infection and the way of colonisation of other organs via macrophages. In other cases, we also observed the microorganisms in the liver (Körting et al. 1994).

Editorial Responsibility: Managing Editor
According to their light microscopical observation of schizogony and sporogony, Jirovec et al. (1947) assigned the microorganisms to the Haplosporidia. We suggest relating the microorganisms to the bacteria since no nucleus appeared ultrastructurally. Furthermore, the central structure of the agent seems to be similar to that of a bacterium. However, all attempts to cultivate these organisms have until now remained without success (Körting et al. 1994). Whether the formation of intracytoplasmic vacuoles is a reaction of the host cell to the agent or a part of this mysterious organism cannot be answered with the available electron micrographs. The strange, radiated surface coat of the organisms may also be a reaction of the host cells. An influence of fixation (e.g. shrinkage) on the formation of these structures during EM preparation cannot be excluded. Furthermore, the meaning of the unusual compartmentalised structures, which are partly extruded, remains unclear.

The origin and transmission of the organisms are enigmatic. The preferred lifestyle of tench, i.e. dwelling in standing water, possibly plays a role in the uptake of the microorganisms, which perhaps originate from the muddy bottoms of the ponds. Mechanical damage to the skin due to dense fish population may be another factor in the route of transmission. The microorganism is evidently a bacterium, however strange and uncultivable it may be, and should no more be designated as a parasite. The name of the bacterium could remain as Sporozoon tincae.

Acknowledgements. We thank Mrs K. Rohn and Mrs E. Nicksch for their technical assistance

\section{LITERATURE CITED}

Jirovec, O., Schäferna, K., Skorpil, F. (1947). Sporozoon tincae, a pathogenic parasite of the tench. Parasitology 38 : $145-149$

Körting, W., Kaup, F.-J., Hermanns, W. (1987). Sporozoon tincae. In: Deutsche Veterinärmedizinische Gesellschaft (ed.) Tagung der Fachgruppe Fischkrankheiten, 28-29 Oktober 1987, München. DVG, Gießen, p. 77-83

Körting, W., Bernoth. E. M., Kaup, F.-J. (1994). Krankheiten der Schleie. In: Deutsche Veterinärmedizinische Gesellschaft (ed.) Tagung der Fachgruppe Fischkrankheiten, 22-23 September 1993, Hannover. DVG, Gießen (in press)

Volf, F., Dvořák, B. (1928). Protozoální nákaza línů. Sbor. Výzkum. Ústaví Zemědělských. 43: 3-23

Manuscript first received: June 7, 1994

Revised version accepted: September 20, 1994 